\title{
Vigilancia de los niveles de zinc protoporfirina (ZPP) y plomo en sangre en trabajadores de una imprenta en Santa Fe de Bogotá
}

\author{
Henry Cuevas ${ }^{1}$, Jaime Ortiz ${ }^{2}$, Fernando de la $\mathrm{Hoz}^{1}$, Jorge Raad ${ }^{3}$
}

\begin{abstract}
Resumen
Se estudiaron las concentraciones de zinc protoporfirina (ZPP) en sangre en un grupo de 252 trabajadores de una imprenta y se midieron las concentraciones de plomo en sangre $(\mathrm{PbB})$ en 52 de estos trabajadores. Veintidós trabajadores tenían niveles sanguíneos de ZPP mayores de $70 \mathrm{mg} / \mathrm{dL}$. Solamente un trabajador tuvo niveles de plomo en sangre levemente elevados ( $39 \mathrm{mg} / \mathrm{dL}$; punto de corte: $38 \mathrm{mg} / \mathrm{dL}$ ). Aunque estos trabajadores realmente no estuvieron expuestos ocupacionalmente al plomo, sí tenían una baja utilización de elementos de protección personal. El análisis encontró una baja tasa de negativos falsos para ZPP $(0,3 \%)$ y un alto valor predictivo negativo a un umbral de $38 \mathrm{~m} / \mathrm{dL}$ de plomo. Para este tamizaje con ZPP, se calculó un ahorro del $69,7 \%$ del costo de los análisis de plomo en sangre. Se recomienda el uso de la ZPP como un método económico de tamizaje y sugerimos la determinación de plomo en sangre $(\mathrm{PbB})$ para niveles mayores de $70 \mathrm{mg} / \mathrm{dL}$ de $\mathrm{ZPP}$.
\end{abstract}

\section{Summary}

The concentrations of zinc protoporphyrine (ZPP) in the blood of 252 workers from a printshop were studied and the lead concentration in blood $(\mathrm{PbH})$ in 52 workers was measured. Twenty-two workers had blood levels higher than $70 \mathrm{mg} / \mathrm{dL}$. Only one worker had a slightly high blood-lead level ( $39 \mathrm{mg} / \mathrm{dl}$; cut-off point $38 \mathrm{mg} / \mathrm{dL}$ ). Even though these workers were not really exposed to lead in their daily work, there was little use of personal protection equipment. Analysis found a low rate of ZPP false negatives (0.3\%) and high negative predictive values for a threshold of $38 \mathrm{mg} \mathrm{Pb} / \mathrm{dL}$. Using this ZPP screening, it is calculated that a $69.7 \%$ saving of the blood-lead analysis cost can be made. The use of ZPP is thus recommended as being an economical screening process and useful for the determination of lead in blood (PhB) for levels higher than $70 \mathrm{mg} \mathrm{ZPP/dL}$.

Aunque existen muy pocos informes epidemiológicos de exposición a plomo en trabajadores de imprentas, la literatura en salud tiene diversos estudios de exposición a plomo ocupacional en diferentes tipos de industrias (1-3).

Algunos de estos análisis epidemiológicos de plomo en sangre y fuentes ambientales de exposición han enfatizado la fuerte relación existente entre el plomo en aire y sus niveles sanguíneos y aunque un porcertaje relativamente bajo de plomo ingerido es absorbido, los hábitos pobres de higiene perscnal por parte de los trabajadores de la industria también se consideran una fuente potencial en la ingestión no intencional que contribuye al aumento de los niveles de plomo sanguíneo (4).

\footnotetext{
División Centros de Control de Enfermedades, Instituto Nacional de Salud, Santa Fe de Bogotá.

2 Laboratorio de Sanidad del Ambiente, Instituto Nacional de Salud, Santa Fe de Bogotá.

3 Subdirección de Epidemiología y LNR, INS; Universidad de Caldas, Manizales.
} 
Para fines de monitorización biológica, la exposición crónica a plomo se mide mediante indicadores de dosis interna como el plomo en sangre e indicadores de efecto como la zinc protoporfirina. La exposición crónica y sus efectos se miden a través de metabolitos tales como la zinc protoporfirina (ZPP) que, además de acumularse en los eritrocitos como resultado de la absorción crónica de plomo o en anemias por deficiencia de hierro, su determinación es de una gran simplicidad técnica y de bajo costo (5).

Debido a que algunos efectos clínicos han sido correlacionados con niveles elevados en sangre tanto de plomo como de ZPP, la monitorización de este elemento y compuesto facilita la identificación de aquellos individuos que pueden estar en riesgo de tener niveles elevados de plomo corporal (6).

En el presente estudio, se determinaron los valores de ZPP en los trabajadores de una imprenta en Santa Fe de Bogotá, expuestos a concentraciones no conocidas de plomo ocupacional.

\section{Materiales y métodos}

Para el presente estudio descriptivo, de los 280 trabajadores de la imprenta, se tomaron 252 muestras a empleados enviados por la empresa para vigilancia de niveles de ZPP y plomo en sangre, y que que accedieron a participar en forma voluntaria, los cuales se desplazaron al Laboratorio de Sanidad del Ambiente y al Laboratorio Nacional de Referencia del Instituto Nacional de Salud, para el análisis de ZPP en sangre y el diligenciamiento de una encuesta previamente elaborada.

Se tomaron $7 \mathrm{~mL}$ de sangre la cual se analizó para ZPP, utilizando un hematofluorómetro AVIV-modelo 206; se consideró como punto de corte a $70 \mu \mathrm{g} / \mathrm{dL}$, a partir del cual la ZPP se considerada elevada (7).

Se acordó con el coordinador de salud ocupacional de la imprenta, que aquellas muestras que presentaran valores mayores o iguales a 50 $\mu \mathrm{g}$ de ZPP/dL, se les realizara determinación directa de plomo por espectrofotometría. De las muestras restantes, se seleccionaron al azar 26 para ser leidas por espectrofotometría de absorción atómica horno de grafito (determinación directa de plomo en sangre) utilizando un espectrofotómetro 2380-horno de grafito HGA400 (Perkin-Elmer) y automuestrador AS-40 (Perkin-Elmer). Se consideraron valores normales en la población general hasta de $38 \mu \mathrm{g} / \mathrm{dL}$.

A través de una encuesta efectuada por una persona capacitada del Grupo de Sanidad del Ambiente, se indagó por información epidemiológica básica y otros factores asociados a la exposición con plomo tales como: tiempo de trabajo en la empresa, utilización de elementos de protección, sección en la cual trabaja y actividad que realiza en la empresa.

Para efectos del análisis y con base en la organización de la imprenta, se dividió la empresa en 13 secciones y el desempeño de los trabajadores en 25 actividades.

De acuerdo con criterios estandarizados, se consideró la siguiente clasificación de riesgo ocupacional en:

1. Según sección de trabajo en: exposición alta: personal de las secciones de prensas, armada, impresión y offset; exposición baja: personal de arte y composición, fotomecánica, personal de corrección, encuadernación, áreas admistrativa, financiera y comercial.

2. Según actividad laboral en: exposición alta: para capturador, prensita y mecánico; exposición baja: los demás oficios tanto de las áreas de producción como de administración.

\section{Resultados}

El promedio de edad de la población de trabajadores (252) estudiados fue de 38,5 años, los cuales oscilaron entre 16 y 65 años, con un $40,1 \%$ (101) entre 30 a 39 años de edad (cuadro 1). El $36,5 \%$ (92) fueron mujeres y el $63,5 \%$ (160) hombres. El tiempo promedio de antigüedad de los trabajadores en la imprenta fue de 10,5 años con un mínimo de 10 meses y un máximo de 26 años (cuadro 2).

El $17,1 \%$ (43) de los trabajadores pertenece a secciones consideradas de exposición ocupacional relativamente alta a plomo y $19,5 \%$ 
(49) se desempeña en actividades consideradas de exposición relativamente alta dentro de la imprenta.

El $45,6 \%$ (115) de los trabajadores tenía menos de 10 años de trabajo en la imprenta y $54,3 \%$ (137) lleva con la imprenta 10 años o más. Un $26,2 \%$ de la población total lleva entre 10 y 14 años de trabajo.

La encuesta reveló que solamente el $48 \%$ (98) de los trabajadores considerados con actividad laboral de exposición baja, utilizaba ropa de trabajo adecuada. Un $9,8 \%$ (23) usaba mascarillas y $10,7 \%$ (23) utilizaba guantes. Entre las actividades consideradas de exposición alta, $91,8 \%$ (45) de los trabajadores usaba uniforme. Un $28,5 \%$ (14) utilizaba mascarillas de los cuales el $64,2 \%$ (9) las usaba ocasionalmente. El $65 \%$ (32) utilizaba guantes de los cuales el $68,7 \%$ (22) los usaba ocasionalmente.

Los niveles de ZPP sanguíneos en estos trabajadores variaron entre $12,0 \mu \mathrm{g} / \mathrm{dL}$ y $401 \mu \mathrm{g} /$ dL. La media de ZPP de toda la población analizada fue de $40,0 \mu \mathrm{g} / \mathrm{dL}($ IC $95 \%=38,4-41,5)$,

Cuadro 1. Distribución por edades de los trabajadores de una imprenta en Santa Fe de Bogotá, Colombia.

\begin{tabular}{cccc}
\hline Grupo de edad & Frecuencia & Porcentaje & \% acumulado \\
\hline $16-19$ & 1 & 0,4 & 0,4 \\
$20-29$ & 40 & 15,9 & 16,3 \\
$30-39$ & 101 & 40,0 & 56,3 \\
$40-49$ & 76 & 30,2 & 86,5 \\
$50-59$ & 31 & 12,3 & 98,8 \\
$60-69$ & 3 & 1,2 & 100,0 \\
Total & 252 & 100,0 & - \\
\hline
\end{tabular}

Cuado2. Duración de la exposición ocupacional en trabajadores de una imprenta en Santa Fe de Bogotá, Colombia.

\begin{tabular}{lrrr}
\hline $\begin{array}{l}\text { Tiempo de } \\
\text { exposición (años) }\end{array}$ & Frecuencia & Porcentaje & $\begin{array}{r}\text { Porcentaje } \\
\text { acumulado }\end{array}$ \\
\hline $0-5$ & 64 & 25,4 & 25,4 \\
$6-10$ & 75 & 29,8 & 55,2 \\
$11-15$ & 58 & 23,0 & 78,2 \\
$16-20$ & 37 & 14,7 & 92,9 \\
$21-25$ & 17 & 6,8 & 99,7 \\
26 Y Más & 1 & 0,3 & 100,0 \\
Total & 252 & 100,0 & - \\
\hline
\end{tabular}

teniendo niveles entre 23 y $45 \mathrm{u \mu} / \mathrm{dL}$ de ZPP el $75,4 \%(190)$ de los trabajadores y entre 46 y 68 $\mu \mathrm{g} / \mathrm{dL}$ el $12,7 \%$ (32) (figura 1 y cuadro 3 ).

Del total de 252 muestras analizadas, 4,7\% (12) tuvo niveles de ZPP mayores de $70 \mu \mathrm{g} / \mathrm{dL}$. Un $8,6 \%$ (8) de las mujeres y $2,5 \%$ (4) de los nombres tuvo niveles de $Z P P>70 \mu \mathrm{g} / \mathrm{dL}$ (RP=0,29; IC 95\%=0,09-0,93).

El estudio de la distribución por secciones y oficios de la población de trabajadores con niveles mayores de $70 \mu \mathrm{g} / \mathrm{dL}$ de ZPP, determinó que no existen diferencias significativas por estas categorías.

El $83,3 \%$ de los trabajadores que estaban por encima del punto de corte, llevaban 10 o más años de trabajo con la imprenta $(\mathrm{RP}=4,5$; IC $95 \%=0,95-20,4)$ con una media de 14,5 años. Solamente 2 trabajadores, ambos de la sección de acabados y cuya actividad es el trabajo con las prensas, tuvieron un tiempo de trabajo menor de 10 años. El 66,6\% (80) llevaba entre 10 y 19 años de trabajo con la imprenta.

De los trabajadores con niveles de ZPP $>70 \mu \mathrm{g} /$ $\mathrm{dL}$, solamente el $50 \%$ (5) de los considerados con actividad de exposición baja utilizaba ropa

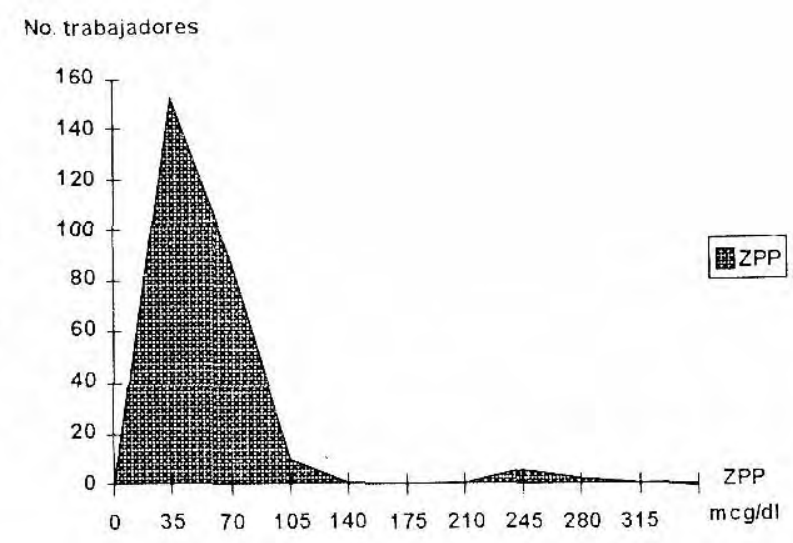

Figura 1. Distribución de ZPP en la población de trabajadores de la imprenta Nacional 
Cuadro 3. Niveles de ZPP mayores de $70 \mathrm{ug} / \mathrm{dl}$ por categorías de oficio entrabajadores de la imprenta

\begin{tabular}{lrrrc}
\hline Oficio & $\mathbf{n}$ & $\%$ & $\begin{array}{c}\text { ZPP } \\
\text { n }\end{array}$ & $\begin{array}{c}\text { 70 mcg/dl } \\
\%\end{array}$ \\
\hline Administrador & 2 & 0,8 & 1 & 50,0 \\
Director & 3 & 1,2 & 1 & 33,3 \\
Auxiliar & 17 & 6,7 & 2 & 11,7 \\
Contador & 17 & 6,7 & - & - \\
Coordinador & 17 & 6,7 & 1 & 5,8 \\
Corrector & 27 & 10,7 & - & - \\
Diagramador & 14 & 5,7 & - & - \\
Digitador & 21 & 8,3 & 2 & 9,5 \\
Encuadernador & 16 & 6,3 & 1 & 6,2 \\
Prensista & 44 & 17,5 & 2 & 4,5 \\
Secretária & 25 & 9,9 & 2 & 8,0 \\
Oficios varios & 8 & 3,2 & 1 & 12,5 \\
otros & 41 & 16,2 & - & - \\
Total & 252 & $\mathbf{1 0 0 , 0}$ & $\mathbf{1 2}$ & 4,7 \\
\hline
\end{tabular}

de trabajo adecuada. Un 10\% (1) usaba mascarilla, pero solo ocasionalmente y el $10 \%$ (1) utilizaba guantes sólo ocacionalmente. De los trabajadores con actividad de exposición alta, $100 \%$ (2) usaba uniforme y guantes ocacionalmente y ninguno utilizaba máscaras de protección.

Se analizaron 52 muestras de trabajadores para determinación directa de plomo en sangre; 44 tenían niveles menores o iguales a $70 \mu \mathrm{g} / \mathrm{dL}$ de ZPP y 12 con niveles de ZPP mayores de 70 $\mu \mathrm{g} / \mathrm{dL}$.

Los niveles sanguíneos de plomo en esta población variaron entre 4 y $39 \mu \mathrm{g}$ de $\mathrm{Pb} / \mathrm{dL}$, con una media de $8,7 \mu \mathrm{g} / \mathrm{dL}$ (IC $95 \%=8,4-8,0)$. La media para mujeres es de $6,5 \mu \mathrm{g} / \mathrm{dL}$ (IC $95 \%=6,1-6,8 ; n=19$ ) con un rango entre 4 y 10 $\mu \mathrm{g} / \mathrm{dL}$; la media en hombres fue de $9.6 \mu \mathrm{g} / \mathrm{dL}$ (IC $95 \%=9,3-9,8 ; n=33$ ) con un rango entre 4 y 39 $\mu \mathrm{g} / \mathrm{dL}$.

Al comparar los niveles de ZPP $<70 \mu \mathrm{g} / \mathrm{dL}$ y plomo en sangre en una curva dosis-respuesta, se obtuvo un coeficiente de correlación de $r=0,04($ IC $95 \%=-0,29-0,30)$. Al tomar los valores de ZPP mayores de $70 \mu \mathrm{g} / \mathrm{dL}$ y compararlos con valores de plomo en sangre, el coeficiente de
Figura 2. Correlación entre valores de Log ZPP y plomo en sangre para valores de $Z P P>70 \mathrm{mcg} / \mathrm{dL}$

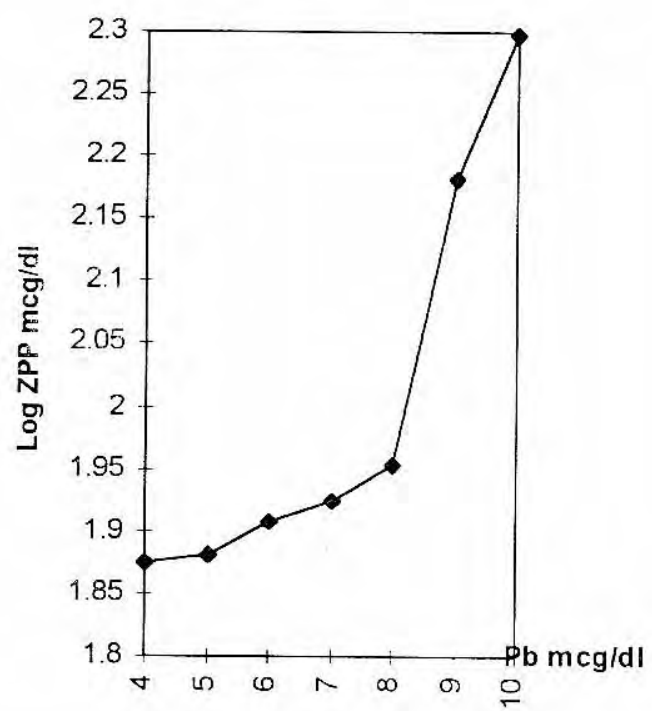

correlación aumenta a $r=0,82$ (IC $95 \%=0,16$ $0,95)$. Al transformar estos valores de ZPP a la escala logarítmica y compararlos con valores de plomo en sangre, se obtiene un coeficiente de correlación de 0,83 (IC 95\%=0,14-0,95) (figura 2).

Sobre la muestra de 52 trabajadores, se obtuvo para la prueba de ZPP una especificidad de $76 \%$ (IC $95 \%=62,2-86,8 \%$ ) con sensibilidad baja (IC $95 \%=0-94,5)$ y con un valor predictivo negativo de $97,5 \%$ (IC 95\%=85,3-99,9\%) y valor predictivo positivo de $0 \%$ (IC 95\%=0-30,1\%). EI porcentaje de positivos falsos para ZPP fue de $4,7 \%$ (12), mientras que sólo se presentó un negativo falso.

\section{Discusión}

Una manera indirecta de medir la exposición crónica a plomo es por medio de la ZPP (zinc protoporfirina) en sangre, la cual se acumula dependiendo de la intensidad y tiempo de exposición (8). Así, la ZPP sirve como una prueba de tamizaje rápida y simple en la determinación de la exposición a plomo (9). El incremento en la concentración de ZPP se detecta en las deficiencias de hierro y como un reflejo de la exposición ocupacional en trabajadores expuestos a plomo (9). 
Los valores altos de ZPP en individuos expuestos a plomo es una fuerte indicación de toxicidad por plomo, pero, este hallazgo no necesariamente indica exposición excesiva a plomo debido a que la anemia por deficiencia de hierro también lleva a elevados niveles de ZPP (10).

El presente estudio es una primera aproximación a la exposición ocupacional por plomo en trabajadores de una imprenta, el cual nos arroja un conocimiento básico de la misma.

En el estudio se obtuvo una media de $40 \mu \mathrm{g} / \mathrm{dL}$ para ZPP y una media de plomo en sangre de $8,7 \mu \mathrm{g} / \mathrm{dL}$ la cual es similar a otros estudios de ZPP realizados en poblaciones no expuestas a plomo $(11,12)$.

El $95,2 \%$ de la población estudiada tenía valores normales de ZPP. De 252 personas, el método de hematoflourometría determinó que 240 personas tenían niveles de ZPP menores de $70 \mu \mathrm{g} / \mathrm{dL}$ lo que nos indica que tales personas no tenían niveles ocupacionales de plomo, como tampoco niveles bajos de hierro sanguíneo.

Al hacer la determinación de plomo en sangre a los restantes 12 trabajadores $(>70 \mu \mathrm{g} / \mathrm{dL}$ ) y quienes potencialmente podrían tener niveles ocupacionales de plomo o deficiencia de hierro, quedó descartado que tuvieran niveles altos de plomo dejando la posibilidad de la deficiencia por hierro. Justamente es en este grupo donde se determinó un numero alto de mujeres con respecto a hombres $(8 ; \mathrm{RP}=0,29)$.

Los anteriores resultados apoyan la idea de que los trabajadores de esta imprenta no son una población ocupacionalmente expuesta a plomo y respaldan el conocimiento de que en las imprentas actuales y con las nuevas tecnologías de impresión, se utilizan esporádicamente volúmenes bajos de plomo en la fundición e impresión de algunos trabajos especiales.

Al comparar los resultados obtenidos por ZPP y plomo en sangre, de las 40 muestras menores de $70 \mu \mathrm{g}$ de $\mathrm{ZPP} / \mathrm{dL}$, se encontró que estos valores son concordantes para las cifras esperadas por cuantificación de plomo (valor predictivo negativo $=97,5 \%$ ).
Ninguno de los individuos con valores de ZPP mayores de $70 \mu \mathrm{g}$ obtuvo niveles de plomo en sangre mayores o iguales a $39 \mu \mathrm{g} / \mathrm{dL}$. Esto se explica por un valor predictivo positivo bajo de la prueba. Estos resultados son similares a los obtenidos en ensayos previos realizados por nuestro laboratorio (11).

La técnica hematoflurométrica utilizada como tamiz en este estudio nos permitió detectar que $95,2 \%$ de la población estudiada tenía niveles normales de plomo, lo que implica una alta especifidad.

Si bien es cierto que la comparación utilizando niveles bajos de ZPP con los niveles encontrados para plomo en sangre no mostraron correlación $(r=0,04)$, al comparar aquellos niveles de ZPP mayores de $70 \mu \mathrm{g} / \mathrm{dL}$ con los niveles de plomo en sangre, encontramos una fuerte correlación $(r=0,82)$. Además, es interesante anotar que en un solo caso se obtuvieron valores de plomo en el límite superior del punto de corte (hasta $38 \mu \mathrm{g} / \mathrm{dL}$ ), con niveles de $39 \mu \mathrm{g} / \mathrm{dL}$ de plomo ( $\mathrm{S}=+/-4 \mu \mathrm{g} / \mathrm{dL}$ ), que se correspondieron con niveles de $52 \mu \mathrm{g} / \mathrm{dL}$ de ZPP, lo cual podría ser un indicio de la variación en la susceptibilidad individual al plomo, tal como se ha informado en la literatura (13).

De otra parte, ningún otro valor de ZPP menor de $70 \mu \mathrm{g} / \mathrm{dL}$ tuvo valores iguales o mayores de $39 \mu \mathrm{g} / \mathrm{dL}$. Este hallazgo apoya previas conclusiones del laboratorio del Instituto Nacional de Salud, que niveles de ZPP de $70 \mu \mathrm{g}$ o menos (con un $95 \%$ de probabilidad) es un buen indicador que sujetos normales tienen valores de plomo sanguíneos menores de $38 \mu \mathrm{g} /$ $\mathrm{dL}$ (11). Lo cual nos permite seguir recomendando que, para valores de ZPP por encima del punto de corte de $70 \mu \mathrm{g} / \mathrm{dL}$, es necesario hacer determinación de plomo en sangre.

Una de las ventajas del uso de la ZPP como un método de tamizaje es su economía en términos de ahorro en trabajo, tiempo y costos en relación con la alternativa de realizar el tamizaje midiendo plomo en sangre (14). Al considerar que el costo de una prueba de ZPP es de US $\$ 5,90$ y que una prueba de $\mathrm{Pb}$ en 
sangre cuesta US $\$ 23$, el ahorro económico de utilizar la ZPP varía entre 25\% (cuando todas las muestras tienen valores positivos de ZPP) y $75 \%$ del costo de los análisis de $\mathrm{Pb}$ (cuando ninguna muestra tiene valores positivos de ZPP).

Para el presente estudio y utilizando la fórmula del diferencial entre el costo total de muestras por $\mathrm{Pb}$ y el costo total de muestras por ZPP, teniendo en cuenta el costo de pruebas adicionales de $\mathrm{Pb}$ (12), se calculó un ahorro de $69,7 \%$ del costo de los análisis de plomo para este tamizaje.

Para el cumplimiento de las leyes en salud ocupacional en el país y sobre todo para aquellas empresas en que los trabajadores están potencialmente expuestos a plomo, se puede aplicar la metodología de ZPP por su especificidad y costos bajos.

De otra parte, se han encontrado niveles relativamente altos de ZPP en trabajadores que por su actividad, aparentemente, tendrían un menor riesgo para la contaminación con plomo, tales como digitadores, directivos y administradores de la imprenta. Esta apreciación se relacionó con el tiempo de permanencia en la empresa, obteniéndose que la mayoría de ellos llevaba laborando en la empresa 10 o más años.

Estos trabajadores con niveles altos de ZPP y en quienes se descartaron niveles de plomo altos, podrían probablemente presentar deficiencias de hierro no detectadas.

De otra parte, cabe destacar la baja regularidad de utilización de elementos de protección personal por parte de los empleados de la imprenta lo que podría señalar que ciertas actitudes y comportamientos por parte del personal requieren de programas de capacitación en cuanto al uso de elementos de protección personal.

Si bien es cierto que se desconoce tanto el ambiente familiar y nutricional de la población en estudio, al igual que otros factores externos a la imprenta, estos resultados descartan la exposición a plomo ocupacional y sugieren probablemente que algunos individuos podrían presentar deficiencias por hierro y que algunos mecanismos no ocupacionales pudiesen jugar un papel de importancia en esta deficiencia.

\section{Referencias}

1. Waller K, Osorio AM, Jones $\mathbf{J}$. Lead exposure in a tank demolition crew: implications for the new OSHA construction lead standard. Am J Ind Med 1994;26:693.

2. Gittleman JL, Engelgau MM, Shaw J, et al. Lead poisoning among battery reclamation worker in Alabama. J Occup Med 1994;36:526.

3. Lilis R, Fischbein A, Diamond $\mathbf{S}$, et al. Lead effects among secondary lead smelter workers with blood lead levels below $80 \mu \mathrm{g} / 100 \mathrm{~mL}$. Arch Env Health 1977;65:256.

4. Chavalitnitikul C, Levin L, Chi Chen L. Study and models of total lead exposures of battery workers. Am Ind Hyg Assoc J 1984;44:802.

5. Grunder FI, Mofitt AE. Evaluation of zinc protoporphyrin in an occupational environment. Am Ind Hyg Assoc 1979;40:686.

6. Kononen D. First-year changes in blood lead and protoporphyrin levels within two groups of occupational lead workers. Am Ind Hyg Assoc J 1991;52: 177.

7. Joselow M, Flores J. Application of the zinc protoporphyrin (ZP) test as a monitor of occupational exposure to lead. Am Ind Hyg Assoc J 1977;38:63.

8. Hryhorczuck J, Hogan M, Mallin $\mathrm{K}$, et al. The fall of zinc protoporphyrin leveis in workers treated for chronic lead intoxication. J Occup Med 1985;27:816.

9. Celis JC, Medina LJ. Determinación de zinc protoporfirina como índice de la contaminación con plomo. Bogotá: Universidad de los Andes, Departamento de Ciencias Biológicas; 1990:26-42.

10. Suga R, Fishchinger A, Knoch F, et al. Establishment of normal values in adults for zinc protopophyrin (ZPP) using hematofluorometer: correlation with normal blood lead values. Am Ind Hyg Assoc J 1981;42:637.

11. Granjean P, Jorgensen P, Viskum S. Temporal and interindividual variation in erytrocite zinc-protoporphyrin in lead exposed workers. Brit $J$ Ind Med 1991;48:254.

12. Stanton N, Gunter E, Parson P, et al. Empirically determined lead-poising screening cutoff of the protoflour-Z hematoflourometer. Clin Chem 1989;35: 2104. 